\title{
FINANCIAL MARKET PREDICTION SYSTEM WITH EVOLINO NEURAL NETWORK AND DELPHI METHOD
}

\author{
Nijolė Maknickiené ${ }^{1}$, Algirdas Maknickas ${ }^{2}$ \\ Vilnius Gediminas Technical University, Sauletekio al. 11, LT-10223 Vilnius, Lithuania \\ E-mails: ${ }^{1}$ nijole.maknickiene@vgtu.lt (corresponding author); ${ }^{2}$ algirdas.maknickas@vgtu.lt \\ Received 16 July 2012; accepted 10 September 2012
}

\begin{abstract}
Use of artificial intelligence systems in forecasting financial markets requires a reliable and simple model that would ensure profitable growth. The model presented in the paper combines Evolino recurrent neural networks with orthogonal data inputs and the Delphi expert evaluation method for its investment portfolio decision making process. A statistical study demonstrates the reliability of the model and describes its accuracy. Capabilities of the model are demonstrated using a trading simulation.
\end{abstract}

Keywords: artificial intelligence, forecasting, investment portfolio, exchange rates, Sharpe ratio.

Reference to this paper should be made as follows: Maknickienè, N.; Maknickas, A. 2013. Financial market prediction system with Evolino neural network and Delphi method, Journal of Business Economics and Management 14(2): 403-413.

JEL Classification: C53, D53, G11, G17.

\section{Introduction}

Artificial intelligence methods have become very important in making financial market predictions. The following elements are of major importance: the selection of the input data, the selection of the forecasting tool, and the correct use of the output data. As investors are searching for profitable growth, they require the development of a stable and reliable forecasting model.

Kimoto et al. (1990) proposed a stock market prediction system with modular neural networks; Wang and Leu (1996) used ARIMA-based neural networks. The application of neural networks to stock market prediction was presented by Kulkarni (1996). The accuracy of the prediction depended on neural networks and the input selection.

Stock prices have also been forecasted using evolutionary systems. Kim and Han (2000) proposed a new hybrid of a genetic algorithm with artificial neural networks. The genetic algorithm not only searches for the optimal or near-optimal solutions of the connection weights in the learning algorithm, but also looks for the optimal or near-optimal thresholds of the feature discretization. Hussan et al. (2007) proposed and implemented a fusion model by combining the hidden Markov model, artificial neural networks (NN), 
and genetic algorithms to forecast the behaviour of financial markets. The weighted average of the predictions was used to forecast stock prices and increase the accuracy of the model. Choudhry and Garg (2008) proposed a hybrid machine learning system based on genetic algorithms and support vector machines for stock market prediction, using the correlation between the stock prices of different companies.

Prediction systems based on neuro-fuzzy sets have also been used to predict financial markets. Ang and Quek (2006) proposed a model, which synergizes the price difference forecast method with a forecast bottleneck-free trading decision model. Chiang and Liu (2008) developed a fuzzy rule based system, where the clustering technique and the simplified and wavelet (Chang, Fan 2008) fuzzy rule based systems were integrated for forecasting. The system proposed by Agrawal et al. (2010) used an adaptive neuro-fuzzy inference system for making decisions based on the values of some technical indicators. Among the various technical indicators available, the system used the weighted moving averages, divergence, and RSI (relative strength index). In the paper (Quek et al. 2011), a novel stock trading framework based on a neuro-fuzzy associative memory architecture was proposed. The architecture incorporated the approximate analogical reasoning schema to resolve the problem of discontinuous responses and inefficient memory utilization with uniform quantization in the associative memory structure.

Suppose it is known that $p$ is an element of some set of distributions $P$. Choose a fixed weight $\omega_{q}$ for each $q$ in $P$ such that the $\omega q$ add up to 1 (for simplicity, suppose $P$ is countable). Then construct the Bayesmix $M(x)=\sum_{q} \omega q q(x)$, and predict using $M$ instead of the optimal but unknown $p$. How wrong could this be? The recent work of Hutter provides general and sharp loss bounds (Hutter 2001): Let $L M(n)$ and $L p(n)$ be the total expected unit losses of the $M$-predictor and the $p$-predictor, respectively, for the first $n$ events. Then $L M(n)-L p(n)$ is at most of the order of $\sqrt{ } L p(n)$. That is, $M$ is not much worse than $p$. And in general, no other predictor can do better than that. In particular, if $p$ is deterministic, then the $M$-predictor will not make any more errors. If $P$ contains all recursively computable distributions, then $M$ becomes the celebrated enumerable universal prior. The aim of this paper is to construct a model that could make predictions with a small enough difference $M(t)-p(t)$ for some fixed time $t$.

Schmidhuber et al. (2005) introduced a general framework of sequence learning algorithms, EVOlution of recurrent systems with LINear outputs (Evolino). Evolino uses evolution to discover good recurrent neural network hidden node weights, while using methods such as linear regression or quadratic programming to compute the optimal linear mappings from the hidden state to the output. In some cases, quadratic programming is used to maximize the margin. Evolino-based Long Short-Term Memory (LSTM) can solve tasks that Echo State nets cannot.

The block diagram of an LSTM recurrent neural network is shown in Figure 1.

The Evolino recurrent neural network forms an LSTM network with $N=4 n$ memory cells, where $N$ is the total number of neurons and $n$ is the number of memory cells. The genetic evolution algorithm is applied to each quartet of memory cells separately. The cell has an internal state $S$ together with a forget gate $(G F)$ that determines how much the state is attenuated at each time step. The input gate $(G I)$ controls access to the cell 
by the external inputs that are summed into the unit, and the output gate $(G O)$ controls when and how much the cell fires. The dark nodes represent the multiplication function and the linear regression Moore-Penrose pseudoinverse method is used to compute the output (light blue circle). A detailed description of the Evolino RNN algorithm can be found in (Schmidhuber et al. 2005, 2006).

Input selection is always important for adapting artificial intelligence systems to forecasting. In the paper by Maknickas and Maknickiene (2012), a statistical research of the orthogonality of inputs was made. The tools of financial prediction were found by searching for dependencies between the time series of various financial indicators or for series that have been exploited.

Suppose

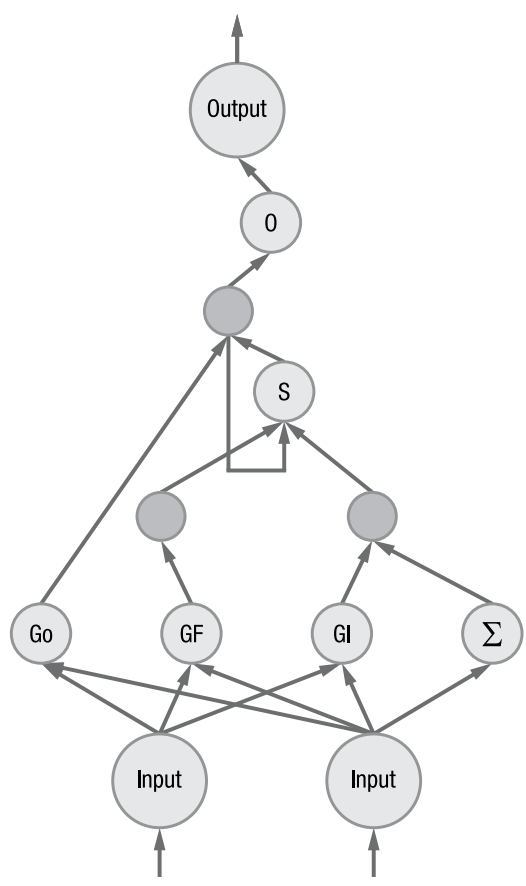

Fig. 1. LSTM network

where the absolute value of the scalar multiplication of vectors $\varepsilon$ describes the degree of orthogonality (true orthogonality cannot be reached for the time series of financial markets). The prediction of one time series output has been obtained by two mostly orthogonal time series inputs.

The output of one part of an AI system can be the input for another part. Some outputs can describe the distribution of predictions and can be analysed. We chose the method applied to expert panel opinions.

The Delphi method was examined in detail by Dalkey (1969). Its use of a panel's opinion has many different modifications. One of them, the fuzzy Delphi, was used for sales forecasting by Chang and Wang (2006) and integrated with artificial NN for stock market forecasting by Kuo et al. (1996).

Trading in a foreign exchange market is not only related to profitability but also to the investment risk. Therefore, in order to diversify the risk, it is necessary to create an investment portfolio. The portfolio optimization problem and optimal portfolio selection methods are considered in (Rutkauskas 2005; Rutkauskas, Stasytyte 2008; Rutkauskas et al. 2009a, 2009b).

The aims of our article are to present the Evolino recurrent neural network prediction model, coordinated and tailored to profitably trade in the currency market, taking investment risk into account. This article focuses more on the model and its reliability and accuracy; it also aims to show the wide scope of investment opportunities. A good forecasting tool will allow focusing more on research and justification of economic processes. 


\section{Forecasting tools}

\subsection{Delphi method}

The Delphi method is based on the assumption that group judgements are more valid than individual judgements. Our observations on the Evolino recurrent neural network prediction (Rutkauskas et al. 2010) made it clear that some of the predictions are very accurate, while some others are contradictory, unstable, and must be rejected. The Delphi method makes it possible to achieve a certain consensus or clustering of forecasts. The steps of classical Delphi method are:

1) The group of experts receives a questionnaire and expresses their prognoses using numeric values, argues their assessments, and completes the questionnaire.

2) The answers are arranged in the ascending order and the media $\mathrm{Me}$ and quartiles $Q_{1}, Q_{3}$ are calculated. After determining the upper and lower quartiles, the range between the two averages $Q_{1} M e$ and $Q_{3} M e$ is considered the most desirable interval. The compatibility of the predictions is calculated, such as whether there is a consensus of the experts. The experts are then familiarised with the results and the arguments and prognoses are made again.

3) The second step is then repeated. Theoretically, the Delphi process can be continuously iterated until a consensus is reached. In practice, the number of iterations is limited by the time available for decision making.

In our method, experts are not people and they can't argue, but several Evolino recurrent neural networks can make predictions and the compatibility of their prediction can be calculated.

\subsection{Compatibility of neural net predictions}

The expert group evaluation of the performance can be considered sufficiently reliable only if the expert evaluation possesses good compatibility of the responses. Therefore, it is necessary to assess the compatibility of the expert assessments and calculate the interquartile coefficient. The variation of the responses is taken to be the difference between the first and third quartiles, Q3-Q1. The interquartile coefficient is the quotient of the variation response by the median:

$$
q=\frac{\mathrm{Q} 3-\mathrm{Q} 1}{M e} .
$$

The interquartile coefficient ranges from 0 to +1 and is close to zero when the distribution has very little variation. In neural network assessment, as in human-expert evaluations, one expert's estimates may be better than that of the group as a whole.

\subsection{Reliability of forecasting}

Time series methods use historical data as their basis. Suppose that, for $t=1, \ldots, N, y(t)$ is the actual value at time $t, \hat{y}(t)$ is the forecast value at time $\mathrm{t}$, and then $\varepsilon(t)$ is the forecast error at time $t$, where $e(t)=y(t)-\hat{y}(t)$. With the purpose of testing the reliability of the model, we will calculate Pearson's correlation coefficient $r$ between $y(t)$ and $\hat{y}(t)$ : 


$$
r=\frac{\sum_{t=1}^{N}(y(t)-\hat{y})(\hat{y}(t)-\hat{y})}{\sqrt{\sum_{t=1}^{N}(y(t)-\hat{y})^{2}} \sqrt{\sum_{t=1}^{N}(\hat{y}(t)-\hat{y})^{2}}} .
$$

This coefficient will help verifying the accuracy and reliability of the model.

\subsection{Profitability and risk of financial forecasting}

We assume that the individual investor, having a certain initial capital, wants to invest in the instruments available for a particular period $T$. Investing the initial capital of $V_{0}$ in a set of instruments, the investor shapes an investment portfolio $p$. Suppose that at the end of the selected period, the investor will realize the entire investment portfolio, in other words, liquidate it into cash, the amount of which will be denoted by $V_{1}$. The purchase and liquidation will be at market prices, which depend on the interaction of the supply and demand in the market. Naturally, the investor wants to maximize the final value of profitability of the invested funds (Rutkauskas 2006) during the period $T$, which is determined by the following formula:

$$
r=\frac{V 1-V 0+D}{V_{0}},
$$

where $D$ is the current income of the portfolio for the period $T$. There are many options for choosing the investment portfolio, but we will explore only three: 1) conservative even distribution of risk; 2) moderate - the objective of the optimal profit with reasonable risk; 3) aggressive, in order to maximize profits with low risk. The most referenced risk/return measures used in finance are the standard deviation and the Sharpe ratio:

$$
S(x)=\frac{r_{x}-r_{f}}{\sigma_{x}},
$$

where $x$ is the investment, $r_{x}$ is the average rate of return of $x, r_{f}$ is the best available rate of return of a risk-free security, and $\sigma_{x}$ is standard deviation of $r_{x}$.

The risk levels of the strategies should be proportional to their Sharpe ratios. Strategies with zero predicted Sharpe ratios should be ignored. Those with positive ratios should be 'held long', and those with negative ratios 'held short'. If strategy $\mathrm{X}$ has a positive Sharpe ratio that is twice as large as that of strategy $\mathrm{Y}$, twice as much risk should be taken with X as with Y. The overall LSTM network scale of all the positions should, in turn, be proportional to the investor's risk tolerance (Sharpe 1994).

\section{Basic architecture and simulation of the prediction algorithm}

During the initial model testing stage, we have given a lot of attention to prediction of various market parameters. We studied stock prices, indexes, various resource prices, and chose a narrow area of research, namely, exchange rate forecasting.

The accuracy of prediction was investigated with the python program using the following steps: 
Data step. Getting historical data on financial markets from Meta Trader-Alpari. For prediction, we chose EUR/USD (Euro and American Dollar), EUR/JPY (Euro and Japanese Yen), USD/JPY (American Dollar and Japanese Yen), EUR/CHF (Euro and Swiss Franc) exchange rates and their historical data for the first input, and for the second input, two years historical data for XAUUSD (gold prices in American dollars), XAGUSD (silver prices in American dollars), QM (Oil prices in American dollars), and QG (gas prices in American dollars). At the end of this step, we had the basis of historical data.

Input step. The python script calculated the ranges of orthogonality of the last 80-140 points of the exchange rate historical data chosen for prediction, and an adequate interval from the two years historical data of XAUUSD, XAGUSD, QM, and QG. A value closer to zero indicates higher orthogonality of the input base pairs. Eight pairs of data intervals with the best orthogonality were used for the inputs to the Evolino recurrent neural network.

Prediction step. Eight Evolino recurrent neural networks made predictions for a selected point in the future. At the end of this step, we had eight different predictions for one point of time in the future.

Consensus step. The resulting eight predictions were arranged in the ascending order, and then the median, quartiles, and compatibility were calculated. If the compatibility was within the range [0;0.024], the prediction was right. If not, then step 3 was repeated, sometimes with another 'teacher' if the orthogonality was similar. At the end of this step, we had one most probable prediction for the chosen exchange rate.

Investment portfolio step. Repeating steps 1-4 for the other exchange rates allowed having a set of exchange rate forecasts and building an investment portfolio. The first portfolio was made from the four exchange rates (EUR/USD, EUR/JPY, USD/JPY, EUR/CHF), and the investment amount was divided equally at every step of the investing. The second portfolio was made from the four same exchange rates but the amount invested was divided by the projected percentage gain. The third choice of investment portfolio consisted of that exchange rate whose projected growth rate was the highest. The basic architecture of the prediction algorithm is shown in Figure 2.

With the aim of verifying the accuracy and reliability of the model predictions, a statistical analysis of the model was carried out. The validation of the prediction was measured by the correlation between the predicted values and the real values in the future. Different exchange rates in the first input and different historical data in the second input guaranteed a random selection of data inputs for the model. Figure 3 shows the distribution of 200 Pearson's correlation coefficients.

All tests were made in the time period from 12/2011 to 06/2012. The choice of the period was not specifically planned.

Values of the correlation coefficient equel 1 were received in $30 \%$ of all tests, which means an excellent prediction. Correlation coefficient within the range $[0.6 ; 1]$ was $68 \%$ of all tests, which means a very good prediction, and within the range $[0 ; 1]-77 \%$, which means that it is a very good predictor of the direction of change, which is very important for investors as well. And only $23 \%$ negative correlation coefficients were received in the statistical research of the model. 


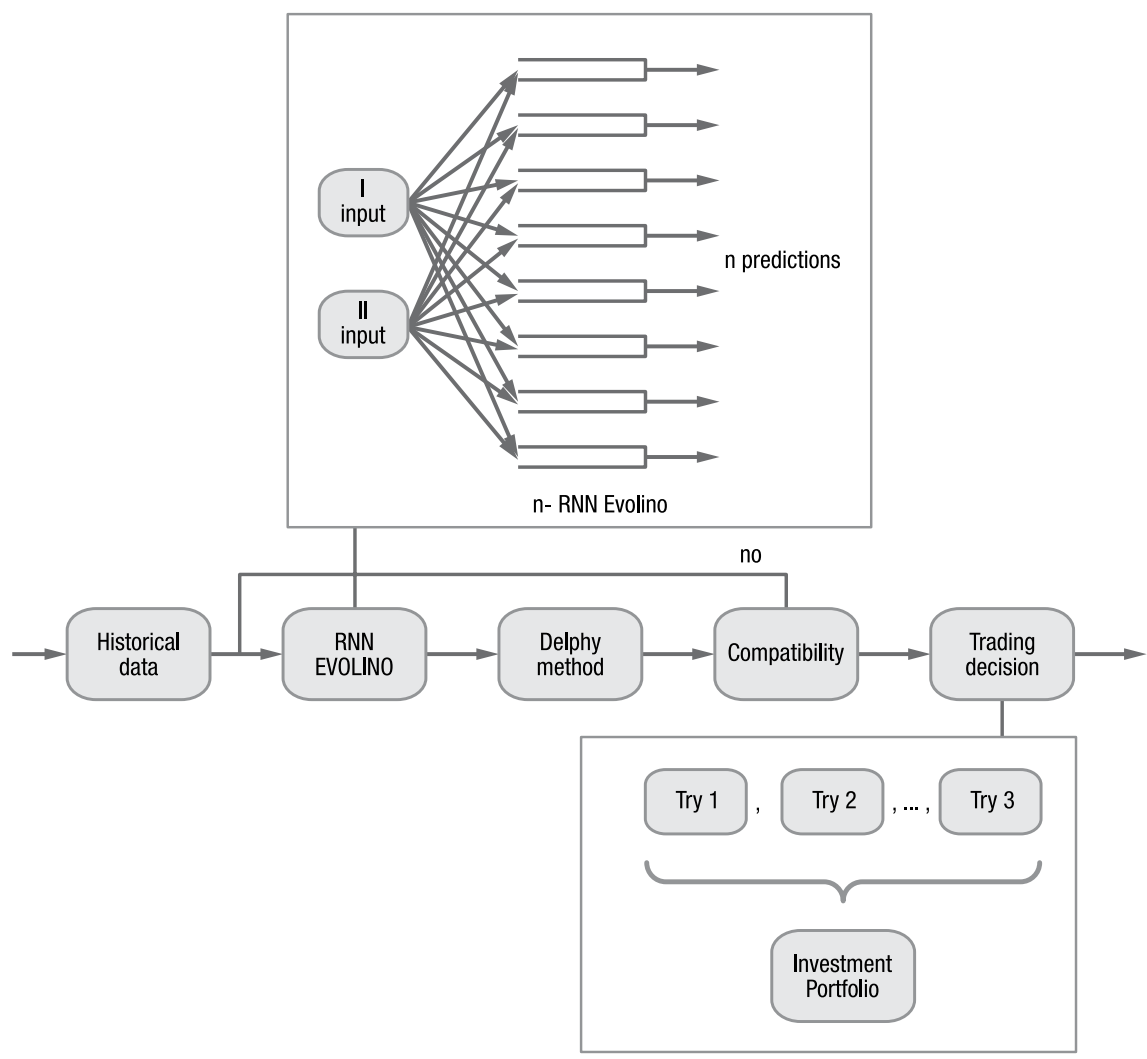

Fig. 2. Scheme of the model

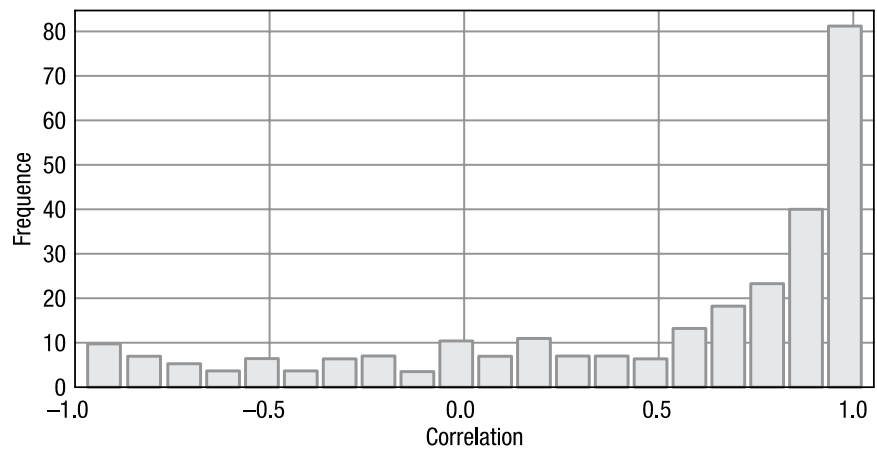

Fig. 3. Statistical analysis of the model's accuracy (200 tests)

\section{Prediction simulation}

Having several different exchange rate forecasts allows an investor to choose different investment portfolios and reduce the investment risk, thus increasing its reliability. Three investment portfolios have been tested: 
Conservative. The first portfolio was made from four exchange rates (EUR/USD, EUR/ JPY, USD/JPY, EUR/CHF) with the investment amount divided equally at every step of investing (3 days in our research). The investor, having four predictions from the model, can choose one from the three operations: buying - if the exchange rate increases, selling - if the exchange rate decreases, and keeping - if the prediction has some doubt, such as a very high variation. Every operation with exchange rates has some damage, which equals to 0.02 of the operation.

Moderate. The second portfolio was made from the same four exchange rates. The investor, having four predictions from the model, in order to maximize profits, divided the initial investment amount by the projected percentage gain.

Aggressive. The third portfolio was made from the same exchange rates but the entire amount was invested in only one exchange rate, which had the greatest predicted profit. The comparison of this portfolio's percentage profit of two variants in the time period 12/2011-06/2012 is shown in Figure 4.

The credibility of the model's forecast, building investment portfolios by the projected gain, increases the profitability of the investment from $12-15 \%$ to $20-25 \%$ and $27-35 \%$ in 40 trading days with the different degrees of risk. The standard deviation describes the portfolio risk and the Sharpe ratio indicates the expected differential return per unit of risk associated with the differential return. The risk free rate was put at $3 \%$ per year. The ratios of the different portfolios are compared in Table 1 . The first column gives names of pairs of different portfolios, and two single currency prediction tests without portfolio for comparison. At the second column are standard deviation averages for the entire period. The third column provides Sharpe ratio averages for the entire period.

After selecting three different levels of riskiness of the investment portfolios, all portfolios had a good positive Sharpe index and the aggressive portfolio had a very good Sharpe index (greater than 1). Using only one exchange rate (EUR/USD and USD/JPY) resulted in a standard deviation of $0.61-0.62$, and a Sharpe ratio of 0.73 . Investments based on the knowledge of the prediction of the recurrent neural network 'team' become more reliable and more profitable. The increased reliability of the model provides a decision maker with big investment opportunities and the freedom of choice.
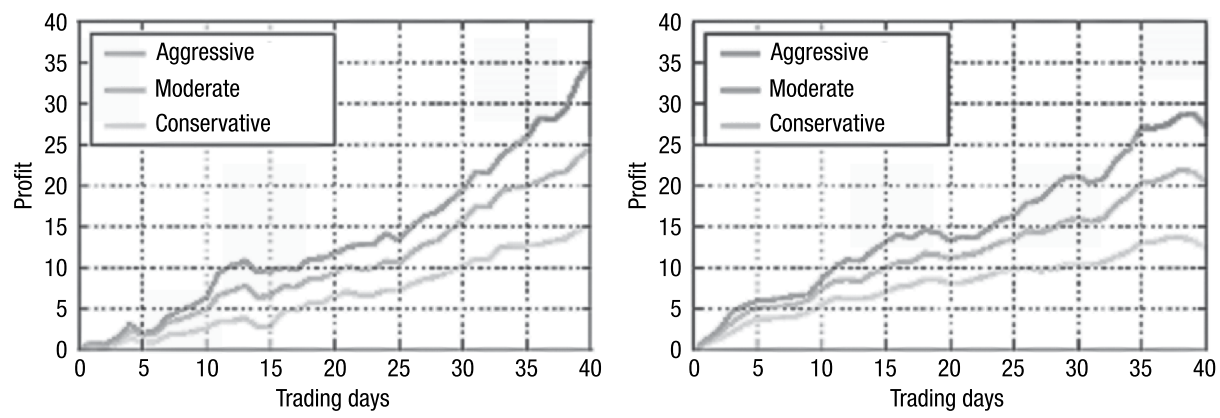

Fig. 4. The percentage of profit growth in two different variants 
Table 1. Portfolio indicators

\begin{tabular}{lcc}
\hline \multicolumn{1}{c}{ Portfolio } & $\begin{array}{c}\text { Standard } \\
\text { Deviation Average }\end{array}$ & $\begin{array}{c}\text { Sharpe } \\
\text { Ratio Average }\end{array}$ \\
\hline Conservative I & 0.32 & 0.78 \\
\hline Conservative II & 0.53 & 0.60 \\
\hline Moderate I & 0.48 & 0.90 \\
\hline Moderate II & 0.63 & 0.80 \\
\hline Aggressive I & 0.71 & 0.81 \\
\hline Aggressive II & 0.72 & 1.07 \\
\hline Without portfolio I & 0.61 & 0.73 \\
\hline Without portfolio II & 0.62 & 0.73 \\
\hline
\end{tabular}

\section{Conclusions}

The model developed, based on the Evolino recurrent neural network and on expert methods, is simple to use and is a good tool for an investor. The reliability of this model, measured by the correlation coefficient, is high enough for profitable trading in the finance market. The credibility of the model's forecast increases the profitability of the investment.

The model allows an investor to make different investment portfolios, based on the choice of different investment strategies with different levels of risk.

This model has a great potential for various investment portfolios and investment strategy choices. It can be easily adapted to trading of other financial indicators or stocks.

\section{References}

Agrawal, S.; Jindal, M.; Pillai, G. 2010. Momentum analysis based stock market prediction using adaptive neuro fuzzy inference system, in Proceedings of the International MultiConference of Engineers and Computer Scientists, vol. 1, March 17-19, 2010, Hong Kong. Newswood Limited, $526-531$.

Ang, K.; Quek, C.; 2006. Stock trading using RSPOP: a novel rough set-based neuro-fuzzy approach, IEEE Trans. Neural Netw. 17(5): 1301-1316. http://dx.doi.org/10.1109/TNN.2006.875996

Chang, P.-C.; Fan, C.-Y. 2008. A hybrid system integrating a wavelet and tsk fuzzy rules for stock price forecasting, IEEE Transactions on Systems, Man, and Cybernetics Part C: Applications and Reviews 38(6): 802-815. http://dx.doi.org/10.1109/TSMCC.2008.2001694

Chang, P.-C.; Wang, Y.-W. 2006. Fuzzy delphi and back-propagation model for sales forecasting in pcb industry, Expert Systems with Applications 30(4): 715-726.

http://dx.doi.org/10.1016/j.eswa.2005.07.031

Chiang, P.; Liu, C. 2008. A tsk type fuzzy rule based system for stock market prediction, Expert Systems with Applications 34(1): 135-144. http://dx.doi.org/10.1016/j.eswa.2006.08.020

Choudhry, R.; Garg, K. 2008. A hybrid machine learning system for stock market forecasting, World Academy of Science, Engineering and Technology 39: 315-318.

Dalkey, N. C. 1969. The delphi method, Tech. Rep. RM-5888-PR. RAND Corporation.

Hussan, M.; Nath, B.; Kirley, M. 2007. Fussion model hmm, ann and ga for stock market forecasting, Experts Systems with Applications 33: 171-180. http://dx.doi.org/10.1016/j.eswa.2006.04.007 
Hutter, M. 2001. General loss bounds for universal sequence prediction, in ICML 2001, Ed. by Morgan Kaufmann. June 28 - July 1, 2001, Williams College, Williamstown, MA, USA. ACE 210-217. ISBN 1-55860-778-1.

Kim, K.; Han, I. 2000. Genetic algorithms approach to feature discretizationin artificial neural networks for the prediction of stock price index, Expert Systems with Applications 19(2): 125-132. http://dx.doi.org/10.1016/S0957-4174(00)00027-0

Kimoto, T.; Asakawa, K.; Yoda, M.; Takeoka, M. 1990. Stock market prediction system with modular neural networks, in International Joint Conference on Neural Networks, vol. 1, June 17-21, 1990, San Diego, CA, USA. IEEE Press, 1-6.

Kulkarni, A. S. 1996. Application of neural networks to stock market prediction, Tech. rep. [online], [cited 12 June 2012]. Available from Internet: www.machine-learning.martinsewell.com

Kuo, R.; Lee, L.; Lee, C. 1996. Integration of artificial neural networks and fuzzy delphi for stock market forecasting, in IEEE International Conference on Systems, Man, and Cybernetics, vol. 2. 14-17 Oct., 1996, Beijing, China. IEEE Press, 1073-1078.

http://dx.doi.org/10.1109/ICSMC.1996.571232

Maknickas, A.; Maknickiene, N. 2012. Influence of data orthogonality to accuracy and stability of financial market predictions, in IJCCI 2012: 4th International Joint Conference on Computational Intelligence, Barcelona, Spain, 5-7 October, 2012. Setubal: INSTICC, 616-619.

Quek, C.; Guo, Z.; Maskell, D. L. 2011. A novel fuzzy associative memory architecture for stock market prediction and trading, International Journal of Fuzzy System Applications 1(1): 61-78. http://dx.doi.org/10.4018/ijfsa.2011010105

Rutkauskas, A. 2005. Portfelio sprendimai valiutu kursu ir kapitalo rinkose, Business: Theory and Practice 6(2): 107-116.

Rutkauskas, A. 2006. Adekvačiojo investavimo portfelio anatomija ir sprendimai panaudojant imitacines technologijas, Ekonomika 75: 52-76.

Rutkauskas, A.; Maknickiene, N.; Maknickas, A. 2010. Approximation of dji, nasdaq and gold time series with evolino neural networks, in The 6th International Scientific Conference Business and Management 2010, May 13-14, 2010, Vilnius, Lithuania. Vilnius: Technika, 170-175.

Rutkauskas, A.; Stasytyte, V. 2008. Stratification of stock profitabilities - the framework for investors' possibilities research in the market, Intellectual Economics 1(3): 67-72.

Rutkauskas, A.; Stasytyte, V.; Stankeviciene, J. 2009a. Profit, riskness and reliability - threedimensional base for investment decisions management, in Modeling and Analysis of Safety and Risk in Complex Systems: Proceedings of the Ninth International Scientific School, July 7-11, 2009, Saint-Petersburg, Russia. MASR, 105-110.

Rutkauskas, A.; Stasytyte, V.; Borisova, J. 2009b. Adequate portfolio as a conceptual model of investment profitability, risk and reliability adjustment to investor's interests, Economics and Management 14: 1170-1174.

Schmidhuber, J.; Gagliolo, M.; Wierstra, D.; Gomez, F. 2006. Evolino for recurrent support vector machines, in European Symposium on Artificial Neural Networks, April 26-28, 2006, Bruges, Belgium. arXiv preprint cs/0512062, 593-598.

Schmidhuber, J.; Wierstra, D.; Gomez, F. F. 2005. Evolino hybrid neuroevolution/optimal linear search for sequence learning, in Proceedings of the 19th International Joint Conference on Artificial Intelligence, July 30 - August 5, 2005, Switzerland: Morgan Kaufmann Publishers Inc, 466-477.

Sharpe, W. 1994. The sharpe ratio, The Journal of Portfolio Management 21(1): 49-58. http://dx.doi.org/10.3905/jpm.1994.409501

Wang, J.; Leu, J. 1996. Stock market trend prediction using arimabased neural networks, in IEEE International Conference on Neural Networks, vol. 44, June 3-6, 1996, Washington DC, USA. IEE Service Center, 2160-2165. 
Nijolė MAKNICKIENĖ. Assistant, Master in Physics at Vilnius University, Quantum Electronics (Dipl.-Phys.) 1986. Recently assistant at the Department of Financial Engineering of Vilnius Gediminas Technical University. Author of 1 peer-reviewed research paper, and 4 contributions to international conferences. Research interests: capital markets, researching chaotic processes by a neural network.

Algirdas MAKNICKAS. Dr, Graduated in Physics at Vilnius University (Dipl.-Phys.) 1986, Dr techn. (Ph.D.) at VGTU in 2009. Recently associate professor at the Department of Information Technologies of Vilnius Gediminas Technical University and senior researcher at the Institute of Mechanical Sciences of Vilnius Gediminas Technical University. Author of 9 peer-reviewed research papers, and more than 15 contributions to international conferences. He has participated in several EU-funded projects. His recent research interests include: theoretical investigation of particulated solids, software engineering, algorithm theory, chaos forecasting theory. 